\title{
Evidence for Cooper pair diffraction on the vortex lattice of superconducting niobium
}

\author{
A. Maisuradze, ${ }^{1,2}$ A. Yaouanc, ${ }^{2,3}$ R. Khasanov, ${ }^{2}$ A. Amato, ${ }^{2}$ C. Baines, ${ }^{2}$ D. Herlach, ${ }^{2}$ R. Henes, ${ }^{4}$ P. Keppler, ${ }^{4}$ and H. Keller ${ }^{1}$ \\ ${ }^{1}$ Physik-Institut der Universität Zürich, Winterthuerstrasse 190, CH-8057 Zürich, Switzerland \\ ${ }^{2}$ Laboratory for Muon-Spin Spectroscopy, Paul Scherrer Institute, 5232 Villigen-PSI, Switzerland \\ ${ }^{3}$ Institut Nanosciences et Cryogénie, SPSMS, CEA and University Joseph Fourier, F-38054 Grenoble, France \\ ${ }^{4}$ Max-Planck-Institut für Metallforschung, Heisenbergstraße 3, D-70569 Stuttgart, Germany \\ (Received 1 March 2013; revised manuscript received 6 October 2013; published 29 October 2013)
}

\begin{abstract}
We investigated the Abrikosov vortex lattice (VL) of a pure niobium single crystal with the muon spin rotation ( $\mu \mathrm{SR}$ ) technique. Analysis of the $\mu \mathrm{SR}$ data in the framework of the BCS-Gor'kov theory allowed us to determine microscopic parameters and the limitations of the theory. With decreasing temperature the field variation around the vortex cores deviates substantially from the predictions of the Ginzburg-Landau theory and adopts a pronounced conical shape. This is evidence of partial diffraction of Cooper pairs on the VL predicted by Delrieu for clean superconductors.
\end{abstract}

DOI: 10.1103/PhysRevB.88.140509

PACS number(s): 74.25.Uv, 74.20.Fg, 74.78.-w, 76.75.+i

The Ginzburg-Landau (GL) theory for superconductors is expressed in terms of an order parameter $\Delta(\mathbf{r})$. While the absolute value of $\Delta(\mathbf{r})$ determines the local superfluid density, its phase gradient is proportional to the local magnetic vector potential. This phase variation leads to the magnetic flux quantization and the formation of a periodic vortex lattice (VL) in type-II superconductors as was predicted by Abrikosov. ${ }^{1}$ The VL field variation is uniquely characterized by two length scales, the magnetic penetration depth $\lambda$ and the coherence length $\xi_{\mathrm{GL}}$. This simple model turned out to be quite successful in describing the behavior of a superconductor in a magnetic field ${ }^{2,3}$ and serves as a basis for data analysis of experiments. ${ }^{4-6}$ However, as was shown theoretically by Delrieu, ${ }^{7}$ the GL model is unable to describe the magnetic response in clean superconductors at low temperatures and close to the upper critical field $B_{\mathrm{c} 2}$.

Soon after the publication of the microscopic Bardeen-Cooper-Schrieffer (BCS) theory for conventional superconductors, ${ }^{8}$ using a Green's function formalism Gor'kov derived the GL equations from the BCS theory. ${ }^{9}$ Based on the Gor'kov's equation Delrieu analyzed the field variation for classical $s$-wave superconductors in the vicinity of $B_{\mathrm{c} 2} \cdot{ }^{7} \mathrm{He}$ found that for clean superconductors the Cooper pairs (CPs) with ballistic trajectories through the vortex cores diffract on the periodic potential of the VL. As a result, in the low-temperature limit close to $B_{\mathrm{c} 2}$ the spatial field variation around a vortex core has a conical shape, rather than the cosine-like GL behavior, and the fields at the minimum and saddle points are interexchanged relative to the GL prediction. Nearly at the same time Brandt came to the same conclusion based on a nonlocal theory of superconductivity. ${ }^{10}$ To observe the effect of diffraction of CPs, the carrier mean-free path $\ell_{\mathrm{mfp}}$ should exceed the intervortex distance, the measurements should be done in the vicinity of $B_{\mathrm{c} 2}(T)$ such that the $\Delta(\mathbf{r})$ gradient is negligible, and the temperature should be low to minimize thermal fluctuations.

Although the theoretical study of the influence of the diffraction of CPs on the field variation was already performed in 1972, it has not been investigated experimentally in detail so far. Early muon spin rotation $(\mu \mathrm{SR})$ experiments revealed a linear high-field tail in the magnetic field distribution
$D_{\mathrm{c}}^{\exp }\left(B^{Z}\right)$, in agreement with the theoretical expectation. ${ }^{11}$ However, as noticed recently, ${ }^{12}$ it occurred at an unexpectedly high temperature. As we note below the temperature stability is crucial in order to minimize experimental artifacts also leading to a linear high-field tail in $D_{\mathrm{c}}^{\exp }\left(B^{Z}\right)$. On the other hand, a $\mu$ SR study of vanadium did not reveal any deviation from the GL theory. ${ }^{13}$ Thus, superconductivity in the clean limit is one of the critical conditions for the observation of the high-field linear tail. Most of the novel high-temperature superconductors (HTSs) are in the clean limit, and the tail should be observed provided the measurements are performed close to $B_{\mathrm{c} 2}$ and at low temperature. Such studies of HTSs still await to be performed.

As a first superconductor to look for the effect of $\mathrm{CP}$ diffraction, we have chosen metallic niobium $(\mathrm{Nb})$, since it is a simple BCS superconductor and pure single crystals are available. It is a type-II superconductor $(\kappa \approx 0.8>1 / \sqrt{2} \simeq$ 0.7 ), and therefore a VL is expected in the bulk when an external field $\mathbf{B}_{\text {ext }}$ larger than the lower critical field is applied. As shown by small-angle neutron scattering, for $\mathbf{B}_{\text {ext }}$ parallel to the crystallographic $\langle 111\rangle$ direction the VL exhibits a simple triangular lattice. ${ }^{14-16}$

Our $\mathrm{Nb}$ sample was a single crystal disk of $13 \mathrm{~mm}$ diameter and $2 \mathrm{~mm}$ thickness with the $\langle 111\rangle$ axis oriented normal to the disk. The samples studied here and in Ref. 11 come from the same batch, so they should be of the same metallurgical quality. The sample is further characterized when discussing its value of $B_{\mathrm{c} 2}(0)$ (see Supplemental Material ${ }^{17}$ ).

The $\mu \mathrm{SR}$ experiments were performed at the Swiss Muon Source $(\mathrm{S} \mu \mathrm{S})$, Paul Scherrer Institute (PSI), Switzerland, using the general purpose spectrometer (GPS) for $T \geqslant 1.6 \mathrm{~K}$ and the low-temperature facility (LTF) for $T \leqslant 1.6 \mathrm{~K}$. A field-cooled procedure was used with $\mathbf{B}_{\text {ext }}$ perpendicular to the sample plane (parallel to the $\langle 111\rangle$ axis). The $\mu$ SR spectra were recorded in the transverse field geometry; i.e., the initial muon spin polarization $\mathbf{S}_{\mu}$ was perpendicular to $\mathbf{B}_{\text {ext }}$. By definition, $\mathbf{B}_{\mathrm{ext}}$ is parallel to the $Z$ axis of the laboratory orthogonal reference frame. With this geometry the field distribution in the bulk of a superconductor can be probed $D_{\mathrm{c}}^{\exp }\left(B^{Z}\right) .{ }^{18}$ We explicitly distinguish $D_{\mathrm{c}}\left(B^{Z}\right)$ from $D_{\mathrm{c}}^{\exp }\left(B^{Z}\right)$, since $D_{\mathrm{c}}\left(B^{Z}\right)$ only stands for the distribution of a perfect VL without crystal disorder. 


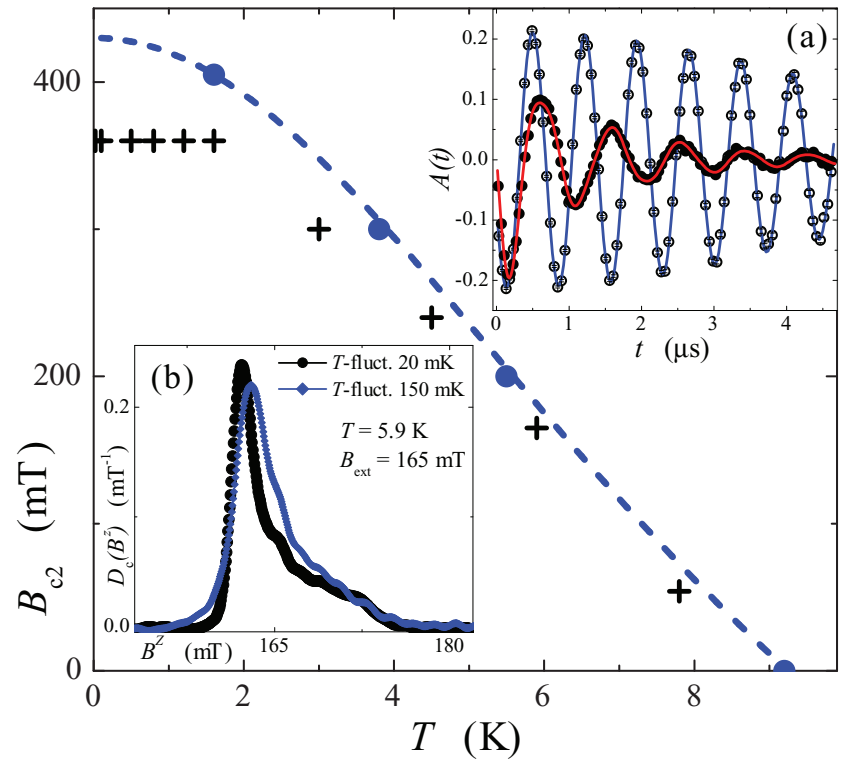

FIG. 1. (Color online) $B_{\mathrm{c} 2}(T)$ for $\mathbf{B}_{\mathrm{ext}} \|\langle 111\rangle$ as determined by $\mu \mathrm{SR}$ measurements for our $\mathrm{Nb}$ single crystal sample (circles). The dashed line corresponds to the equation $B_{\mathrm{c} 2}(T)=B_{\mathrm{c} 2}(0)(1-$ $\left.\tau^{2}\right) /\left(1+\tau^{2}\right)$ proposed in Ref. 19 . Here $\tau=T / T_{\text {co }}$ with $T_{\text {co }}=9.25 \mathrm{~K}$ and $B_{\mathrm{c} 2}(0)=430(2) \mathrm{mT}$. The crosses indicate the points in the field-temperature diagram at which the field distributions displayed in Fig. 2 were measured. Inset (a) shows $\mu$ SR asymmetry spectra $A(t)$ recorded at $1.6 \mathrm{~K}$ in the normal (o) and in the mixed $(\bullet)$ states for $B_{\text {ext }}=450$ and $360 \mathrm{mT}$, respectively. The solid lines are fits of Eq. (1) to the data. The spectra are shown in a rotating frame of 440 and $350 \mathrm{mT}$, respectively. Inset (b) shows the effect of the sample temperature stability on $D_{\mathrm{c}}^{\exp }\left(B^{Z}\right)$. The two measurements were performed at $B_{\text {ext }}=165 \mathrm{mT}$ and $T=5.9 \mathrm{~K}$ with different temperature regulation systems. For one of them the temperature was found to oscillate periodically around an average with a period of a few seconds and a peak-to-peak amplitude of $150 \mathrm{mK}$. For the other system this amplitude was reduced to about $20 \mathrm{mK}$.

The forward and backward positron detectors with respect to $\mathbf{S}_{\mu}$ were used to build the $\mu \mathrm{SR}$ asymmetry time spectra $A(t)$ recorded with total statistics ranging from $1.0 \times 10^{7}$ to $8.0 \times 10^{7}$ positron events. Typical $A(t)$ in the normal and the superconducting states are displayed in inset (a) of Fig. 1. Note that in contrast to the normal state, a strong damping of $A(t)$ in the superconducting state is observed which is characteristic of the local magnetic field variation due to the VL. From these kinds of measurements $B_{\mathrm{c} 2}(T)$ was determined, yielding $B_{\mathrm{c} 2}(0)=430(2) \mathrm{mT}$ (see Fig. 1). This value is smaller than $B_{\mathrm{c} 2}(0)=443 \mathrm{mT}$ reported for a sample with a residual resistivity ratio $\mathrm{RRR}=750 .{ }^{20}$ Hence, for our sample the RRR $>750$. Our value of $B_{\mathrm{c} 2}(0)$ indicates that the sample is of high quality and pure. ${ }^{21}$

As shown in inset (b) of Fig. 1 the temperature stability is important for recording high-quality data close to $B_{\mathrm{c} 2}$. Large fluctuations of temperature may lead to substantial smearing of the measured spectra. The experimental and theoretical field distributions presented in this Rapid Communication were obtained by Fourier transformation (FT) of the Gaussian apodized time spectra (i.e., Fourier transform of $A(t) \exp \left[-\left(t / \sigma_{\text {app }}\right)^{2} / 2\right]$, where $\left.\sigma_{\text {app }}=4.7 \mu \mathrm{s}\right)$. Note that the apodization has no influence on the analysis, since we directly fit $A(t)$, rather than $D_{\mathrm{c}}^{\exp }\left(B^{Z}\right)$.

Figure 2 displays the field distributions measured in the vicinity of $B_{\mathrm{c} 2}(T)$ from a temperature of $7.8 \mathrm{~K}$ close to $T_{\mathrm{c} 0}$ (critical temperature at low field) down to $0.02 \mathrm{~K}$ [see Fig. 1 for the location of the points (crosses) in the field/temperature diagram]. For each $D_{\mathrm{c}}^{\exp }\left(B^{Z}\right)$ measured at LTF a relatively intense sharp peak is present at a field slightly larger than $B_{\text {ext }}$, in contrast to the GPS data for which only a small hump is found. This field structure (sharp peak and small hump) originates from the muons stopped in the cryostat walls and sample holder (background signal). The present GPS data are therefore of a much better quality than previous results obtained in the same temperature range showing an intense background signal. ${ }^{11}$ Qualitatively, a linear high-field tail in $D_{\mathrm{c}}^{\exp }\left(B^{Z}\right)$ is inferred at maybe $1.2 \mathrm{~K}$ and certainly at the lower temperatures, but not at higher temperature. On the other hand, this tail is already seen at $2.6 \mathrm{~K}$ in the published data. ${ }^{11,18}$

A $\mu$ SR spectrum is described by the sum of two contributions:

$$
A(t)=A_{0}\left[F_{\mathrm{s}} R_{\mathrm{s}}(t)+\left(1-F_{\mathrm{s}}\right) R_{\mathrm{bg}}(t)\right],
$$

where $A_{0}$ is the total initial asymmetry and $F_{\mathrm{s}}$ the fraction of muons stopped in the sample. From analysis we determine $A_{0}=0.211$ (2) $[0.217(2)]$ and $F_{\mathrm{s}}=0.993$ (2) [0.817(2)] for measurements carried out with the GPS [LTF] spectrometer. The function

$$
R_{\mathrm{s}}(t)=e^{-\frac{1}{2} \sigma_{\mathrm{s}}^{2} t^{2}} \int D_{\mathrm{c}}\left(B^{Z}\right) \cos \left(\gamma_{\mu} B^{Z} t+\phi_{0}\right) d B^{Z}
$$

describes the time evolution of $\mathbf{S}_{\mu}$ in the sample while $R_{\mathrm{bg}}(t)=$ $\exp \left(-\sigma_{\mathrm{bg}}^{2} t^{2} / 2\right) \cos \left(\gamma_{\mu} B_{\mathrm{bg}}^{Z} t+\phi_{0}\right)$ accounts for the background. Here $\gamma_{\mu}=851.6 \mathrm{Mrad} \mathrm{s}^{-1} \mathrm{~T}^{-1}$ is the muon gyromagnetic ratio, $\sigma_{\mathrm{bg}} \simeq 0.22(2) \mu \mathrm{s}^{-1}$ stands for the background damping, and $\phi_{0}$ is the initial phase. The mean field for the background $B_{\mathrm{bg}}^{Z}$ is only slightly different from $B_{\text {ext }}$ (see Fig. 2). We write $\sigma_{\mathrm{s}}^{2}=\sigma_{\mathrm{nu}}^{2}+\sigma_{\mathrm{dis}}^{2}$, where $\sigma_{\mathrm{nu}}$ accounts for the damping due to the nuclear ${ }^{93} \mathrm{Nb}$ spins, and $\sigma_{\text {dis }}$ is the parameter describing the effect of VL disorder. As usual, we assume the effect of disorder to be modeled by a Gaussian function with a field standard deviation $\sigma_{\text {dis }} / \gamma_{\mu}{ }^{22}$ Although this is a crude approximation, we note that the influence of VL disorder on the high-field tail of $D_{\mathrm{c}}^{\exp }\left(B^{Z}\right)$ is relatively moderate compared to its effect on the low-field side. ${ }^{23}$

We determine $D_{\mathrm{c}}\left(B^{Z}\right)$ from the real space field map $B^{Z}(\mathbf{r})$ of the two-dimensional VL: $D_{\mathrm{c}}\left(B^{Z}\right)=\int_{\text {u.c. }} \delta\left(B^{Z}(\mathbf{r})-\right.$ $\left.B^{Z}\right) d^{2} \mathbf{r}$, where the integral extends over the VL unit cell. In terms of its Fourier components,

$$
B^{Z}(\mathbf{r})=\sum_{\mathbf{K}_{m, h}} B_{\mathbf{K}_{m, h}}^{Z} \exp \left(i \mathbf{K}_{m, h} \cdot \mathbf{r}\right),
$$

where the sum is over the reciprocal space.

First we analyze the data with the numerical solution of the GL (NGL) model for $B_{\mathbf{K}_{m, h}}^{Z}$ using Brandt's method. ${ }^{4}$ It only depends on $\lambda$ and $\xi_{\mathrm{GL}}$. The fit for the highest temperature data is reasonable [see Fig. 2(b)]. We get $\lambda=59.4$ (2) nm and $\sigma_{\mathrm{s}}=0.72(1) \mathrm{mT}$, with $\xi_{\mathrm{GL}}=66.5(2) \mathrm{nm}$ estimated from the measured $B_{\mathrm{c} 2}(7.8 \mathrm{~K})$ and using the $\mathrm{GL}$ formula: $\xi_{\mathrm{GL}}=\left(\Phi_{0} / 2 \pi B_{\mathrm{c} 2}\right)^{1 / 2}$. Here, $\Phi_{0}$ is the flux quantum. As 

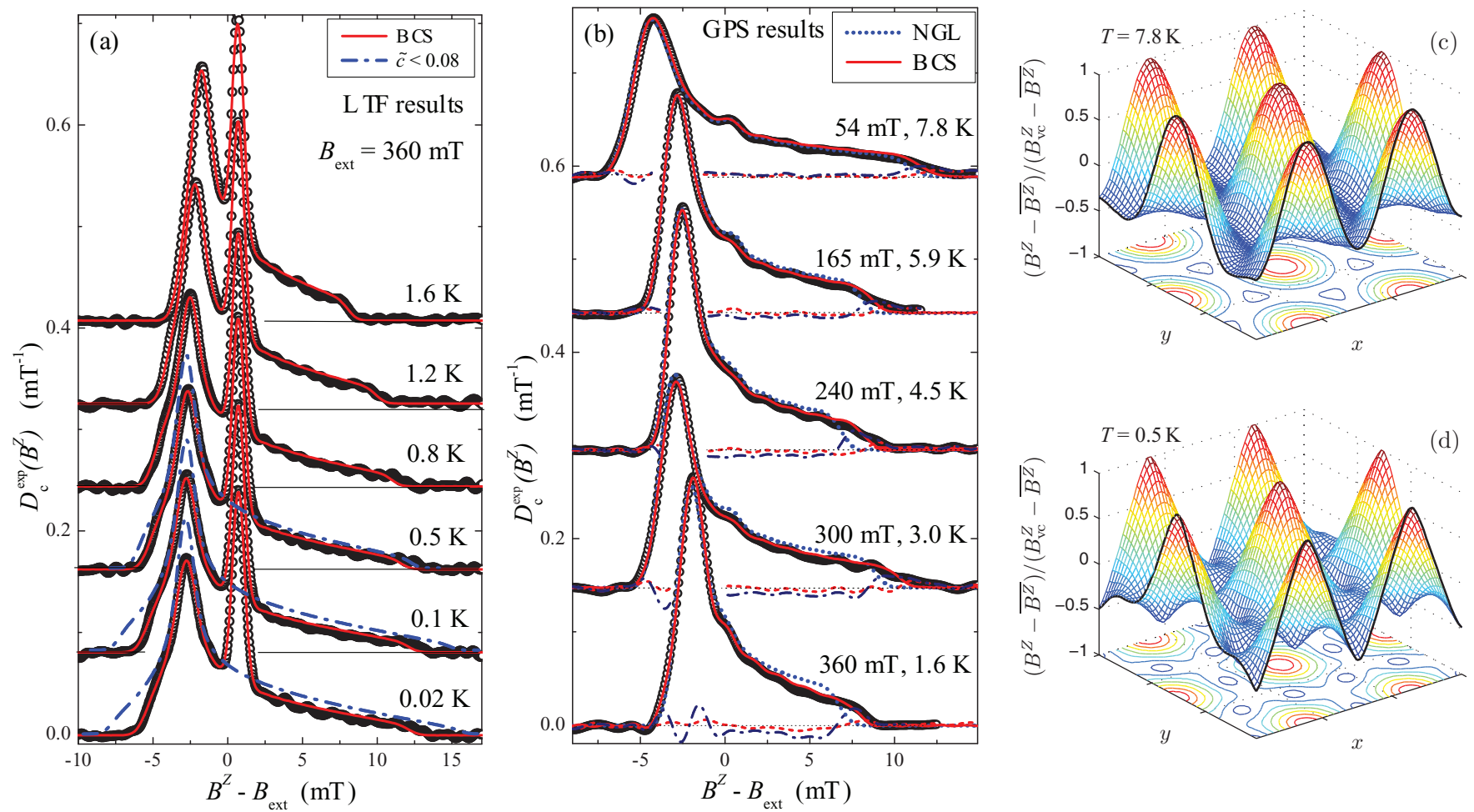

FIG. 2. (Color online) Field distributions $D_{\mathrm{c}}^{\exp }\left(B^{Z}\right)$ of Nb single crystal obtained in the vicinity of $B_{\mathrm{c} 2}(T)$ with the LTF spectrometer (a) and the GPS (b). The solid lines represent best fits of Eqs. (1)-(3) to the data. The fitting parameters are given in Table I of Ref. 17. Leaving the last available parameter $v_{\mathrm{F}}$ free leads to proper fits for $T \geqslant 0.8 \mathrm{~K}$, but not below. The blue dashed-dotted lines in panel (a) represent the results for $T=0.5,0.1$, and $0.02 \mathrm{~K}$. At $0.5 \mathrm{~K}$ the misfits are small, but still present. The solid lines for $T \leqslant 0.5 \mathrm{~K}$ are computed with the fixed value $\tilde{c}=0.08$ for $T=0.6 \mathrm{~K}$. The blue dotted lines in panel (b) correspond to fits with NGL, while the red dashed and blue dashed-dotted lines visualize the differences between $D_{\mathrm{c}}^{\exp }\left(B^{Z}\right)$ and the BCS-Gor'kov and NGL predictions, respectively. Panels (c) and (d) illustrate field variation and contour plot of $B^{Z}(\mathbf{r})$ obtained with BCS-Gor'kov model for the parameters at 7.8 and $0.5 \mathrm{~K}$. $B_{\mathrm{vc}}^{Z}$ denotes the vortex core field.

expected, $\kappa=\lambda / \xi_{\mathrm{GL}}=0.89(1)>1 / \sqrt{2} \simeq 0.7$. The results for $T \leqslant 5.9 \mathrm{~K}$ in Fig. 2(b) were obtained with $\lambda$, $\xi_{\mathrm{GL}}$, and $\sigma_{\mathrm{s}}$ as free parameters. The GL model fails to describe the high-field tails in $D_{\mathrm{c}}^{\exp }\left(B^{Z}\right)$. In addition, unreasonably large $\kappa$ values are derived. For example, $\kappa=48.7 / 28.5=1.7$ at $1.6 \mathrm{~K}$. If $\kappa$ had been taken temperature independent as it should, the misfits would be even worse. As expected, the GL model can only describe $D_{\mathrm{c}}^{\exp }\left(B^{Z}\right)$ very near $T_{\mathrm{co}}$.

Next we analyze the data with the BCS-Gor'kov theory. ${ }^{7,12,24}$ First we discuss the characteristics of $B_{\mathbf{K}_{m, h}}^{Z}$ in the vicinity of $B_{\mathrm{c} 2}(T)$. We use the notations of Ref. 12 . The Fourier component is a function of four parameters: $B_{\mathbf{K}_{m, h}}^{Z}=$ $f_{m, h}(\tilde{a}, \tilde{b}, \tilde{c}, \tilde{d}) .^{7,12,24}$ Here, $\tilde{a}=-\mu_{0} N_{0} \Delta_{0}^{2} \tilde{c} / 2 \overline{B^{Z}}$ does not influence the shape of $B^{Z}(\mathbf{r})$ and therefore $D_{\mathrm{c}}\left(B^{Z}\right)$, but only determines the scale of the field variation. It is proportional to the density of states at the Fermi level $N_{0}$ (per spin, volume, and energy), the quantity $\Delta_{0}^{2}=\overline{|\Delta(\mathbf{r})|^{2}}\left(\overline{|\Delta(\mathbf{r})|^{2}}\right.$ is the spacial average of $\left.|\Delta(\mathbf{r})|^{2}\right)$, and is inversely proportional

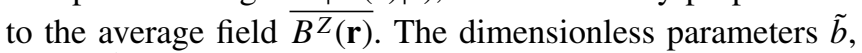
$\tilde{c}$, and $\tilde{d}$ determine the shape of $D_{\mathrm{c}}\left(B^{Z}\right)$ and are expressed by the ratios of four length scales: $\tilde{b}=\left(\Lambda / \pi \xi^{B}\right)^{2}, \tilde{c}=\Lambda / \xi^{T}$, and $\tilde{d}=\Lambda / \ell_{\mathrm{mfp}}$. Here, $\Lambda=\left[\Phi_{0} /\left(2 \pi \overline{B^{Z}}\right)\right]^{1 / 2}$ is a length parameter proportional to the intervortex distance. The field and temperature dependent length scale $\xi^{B}=\hbar v_{\mathrm{F}} /\left(\pi \Delta_{0}\right)$ diverges at $\overline{B^{Z}} \rightarrow B_{\mathrm{c} 2}$, i.e., $\tilde{b} \rightarrow 0$, while $\xi^{T}=\hbar v_{\mathrm{F}} /\left(2 \pi k_{\mathrm{B}} T\right)$.
The parameter $\tilde{c}$ is strongly temperature and field dependent. It vanishes as $T \rightarrow 0$ and diverges at $T \rightarrow T_{\mathrm{c} 0 .}{ }^{12}$ Finally, for clean superconductors $\tilde{d}$ is negligibly small, since $\ell_{\mathrm{mfp}}$ significantly exceeds the intervortex distance.

Cooper pair diffraction may influence $D_{\mathrm{c}}\left(B^{Z}\right)$ when three experimental conditions are met: $\Lambda \ll \ell_{\mathrm{mfp}}, \Lambda \ll \pi \xi^{B}$, and $\Lambda \ll \xi^{T}{ }^{7,12,24}$ The first condition implies a clean superconductor, the second is only satisfied in the vicinity of $B_{\mathrm{c} 2}(T)$, and the third one is only possible at low $T$. Thus, the minimum of $\left\{\ell_{\mathrm{mfp}}, \pi \xi^{B}, \xi^{T}\right\}$ determines the effective diffraction length scale of CPs relative to the intervortex distance $2.693 \times \Lambda$.

The data analysis was done with $\tilde{b} \simeq 0.110(1-b) / b$ fixed $\left[b=\overline{B^{Z}} / B_{\mathrm{c} 2}(T) \simeq B_{\text {ext }} / B_{\mathrm{c} 2}(T)\right] .{ }^{12,17}$ For $\mathrm{Nb}$ we get $0.01<$ $\tilde{b}<0.02$, except for the spectrum at $7.8 \mathrm{~K}$ for which $\tilde{b}=0.04$. Since

$$
\tilde{c}=\frac{\sqrt{\Phi_{0} 2 \pi} k_{\mathrm{B}} T}{\sqrt{\overline{B^{Z}}} \hbar v_{\mathrm{F}}} \simeq \frac{\sqrt{\Phi_{0} 2 \pi} k_{\mathrm{B}} T}{\sqrt{B_{\mathrm{ext}}} \hbar v_{\mathrm{F}}},
$$

the $B_{\mathbf{K}_{m, h}}^{Z}$ depends on $\tilde{a}, \tilde{d}, v_{\mathrm{F}}, T$, and $B_{\text {ext }} \cdot{ }^{17}$

The analysis of $A(t)$ for $T \geqslant 0.8 \mathrm{~K}$ shows that $\tilde{d} \lesssim 0.01$, which agrees with the estimate of $\ell_{\mathrm{mfp}} \simeq 7 \mu \mathrm{m}$ for the $\mathrm{Nb}$ sample with $\mathrm{RRR}=750 .{ }^{20}$ Consequently, we are in the clean limit and $\tilde{d}$ has a negligible influence on $D_{c}\left(B^{Z}\right)$. The results of the analysis are presented in Fig. 2 here and in Table I of Ref. 17. The BCS-Gor'kov model describes the high-field tail 
significantly better at low temperatures while at the highest temperature both models reproduce the data equally well. The deviation from the GL theory and the gradual disappearance of the cutoff singularity at the maximal field is a result of the conical shape of the spatial field variation at the vortex cores [see Fig. 2(d)], which in turn is a consequence of the partial $\mathrm{CP}$ pair diffraction. This deviation cannot be explained by the presence of significant temperature and field fluctuations resulting in a large smearing parameter $\sigma_{\mathrm{s}}{ }^{22}$ If produced artificially the cutoff singularity in $D_{\mathrm{c}}^{\exp }\left(B^{Z}\right)$ at $B_{\mathrm{vc}}^{Z}$ vanishes as is the case in the distribution labeled $150 \mathrm{mK}$ in inset (b) of Fig. 1.17,23 Based on the generalized Bloch equations ${ }^{25-27}$ and the analysis ${ }^{18,28}$ of zero-field $\mu$ SR result we found that the muon diffusion ${ }^{29-31}$ is negligible in the studied $\mathrm{Nb}$ sample (see Ref. 17). A weak pinning ${ }^{17}$ excludes also an influence of the peak effect ${ }^{32-34}$ on the measured field distributions. The BCS-Gor'kov model breaks down for $T \leqslant 0.5 \mathrm{~K}$ as shown by the dashed-dotted lines in Fig. 2(a). A proper description requires us to consider $B_{\mathbf{K}_{m, h}}^{Z}$ as a function of $\tilde{a}$ and $\tilde{c}$ rather than of $\tilde{a}$ and $v_{\mathrm{F}}$, and to keep the value of $\tilde{c}$ at $T=0.6 \mathrm{~K}$ for the lower temperatures [solid lines in Fig. 2(a)]. ${ }^{17}$ This means that the sharpness of the $B^{Z}(\mathbf{r})$ cones is limited by a physical process. Referring to Eq. (3), we suggest that the VL structural disorder may round off the cones, as observed experimentally.

We get $v_{\mathrm{F}}=2.0(2) \times 10^{5} \mathrm{~m} / \mathrm{s}$ from the fits for $T \geqslant 0.8 \mathrm{~K}$. This value is compatible with $v_{\mathrm{F}}=2.73 \times 10^{5}$ and $2.94 \times$ $10^{5} \mathrm{~m} / \mathrm{s}$ determined from magnetization measurements. ${ }^{20,35}$ From the measured $\tilde{a}$ and $\tilde{c}$ we determine the condensation energy $E_{c}=-2 \overline{B^{Z}} \tilde{a} / \mu_{0} \tilde{c}=2 \times N_{0} \Delta_{0}^{2} / 2 .^{2,36}$ While $N_{0}$ is a constant, $\Delta_{0}^{2}$ is field and temperature dependent. At $T=0$ and interpolating $\Delta_{0}^{2}$ to zero field with a conventional formula, ${ }^{12}$ $\left[-2 \overline{B^{Z}} \tilde{a} / \mu_{0} \tilde{c}\right] /\left\{1-\left[\overline{B^{Z}} / B_{\mathrm{c} 2}(0)\right]\right\}=N_{0} \Delta_{0}^{2}(0)$, where $\Delta_{0}(0)$ is the $s$-wave BCS gap which is temperature independent for $T \rightarrow 0 .^{2}$ From $\Delta_{0}(0)=1.45 \mathrm{meV}$ (see Ref. 19) and our estimate for the ground state $E_{c}(0)=N_{0} \Delta_{0}^{2}(0)=2.47(9) \times$
$10^{4} \mathrm{~J} \mathrm{~m}^{-3}$, we obtain $N_{0} \approx 4.6 \times 10^{47} \mathrm{~J}^{-1} \mathrm{~m}^{-3} \mathrm{spin}^{-1} \approx$ $1.3 \mathrm{eV}^{-1}$ atom ${ }^{-1} \operatorname{spin}^{-1}$. Considering the approximate nature of the linear field interpolation, these results are quite close to the specific heat result $N_{0}=0.85 \mathrm{eV}^{-1}$ atom ${ }^{-1} \mathrm{spin}^{-1}$ (see Ref. 37) and the GL condensation energy $B_{\mathrm{c}}^{2} / 2 \mu_{0}=1.6 \times 10^{4}$ $\mathrm{J} \mathrm{m}^{-3}$ for the thermodynamic critical field $B_{\mathrm{c}}=0.20 \mathrm{~T}^{19} \mathrm{In}$ our measurements all the conditions for the observation of partial CP diffraction are met at $T \lesssim 1.2 \mathrm{~K}: \Lambda / \pi \xi^{B} \leqslant 0.11 \ll$ $1, \Lambda / \ell_{\mathrm{mfp}} \lesssim 0.01 \ll 1$, and $\Lambda / \xi^{T}=0.16 \ll 1$.

To conclude, we investigated the magnetic field distribution for the vortex lattice (VL) of a pure $\mathrm{Nb}$ single crystal with the $\mu \mathrm{SR}$ technique. The data were analyzed using the solution of the BCS-Gor'kov equation proposed by Delrieu, ${ }^{7}$ a microscopic description in contrast to the conventional GL picture. As a result, we found strong evidence for partial Cooper pair $(\mathrm{CP})$ diffraction on the periodic potential of the vortex lattice reflected in the conical narrowing of the real space field variation around the vortex cores and in the presence of a high-field linear tail in the field distribution down to $0.02 \mathrm{~K}$, as expected by the BCS-Gor'kov theory. However, the BCS-Gor'kov description is only partially successful as the prediction for the low-field tail at the zero-temperature limit deviates from the experimental observation, presumably due to the residual VL disorder. From the analysis we determined the Fermi velocity $v_{\mathrm{F}}=2.0(2) \times 10^{5} \mathrm{~m} / \mathrm{s}$ and the ground state condensation energy $N_{0} \Delta_{0}^{2}(0)=2.47(9) \times 10^{4} \mathrm{~J} \mathrm{~m}^{-3}$ which are in reasonable agreement with literature results. ${ }^{19,20,35}$ The observation of partial $\mathrm{CP}$ diffraction should not be restricted to $\mathrm{Nb}$. Under proper experimental conditions it should also be seen for any clean type-II superconductor.

This work was performed at the Swiss Muon Source $(\mathrm{S} \mu \mathrm{S})$, Paul Scherrer Institut (PSI), Switzerland, and partly supported by NCCR MaNEP sponsored by the Swiss National Science Foundation.
${ }^{1}$ A. A. Abrikosov, Sov. Phys. JETP 5, 1174 (1957).

${ }^{2}$ M. Tinkham, Introduction to Superconductivity (McGraw-Hill, New York, 1996).

${ }^{3}$ J. B. Ketterson and S. N. Song, Superconductivity (Cambridge University Press, Cambridge, 1999).

${ }^{4}$ E. H. Brandt, Phys. Rev. Lett. 78, 2208 (1997).

${ }^{5}$ J. E. Sonier, J. H. Brewer, and R. F. Kiefl, Rev. Mod. Phys. 72, 769 (2000).

${ }^{6}$ J. E. Sonier, Rep. Prog. Phys. 70, 1717 (2007).

${ }^{7}$ J. M. Delrieu, J. Low Temp. Phys. 6, 197 (1972).

${ }^{8}$ J. Bardeen, L. N. Cooper, and J. R. Schrieffer, Phys. Rev. 108, 1175 (1957).

${ }^{9}$ L. P. Gor'kov, Zh. Eksp. Teor. Fiz. 36, 1918 (1959) [Sov. Phys. JETP 9, 1364 (1959)].

${ }^{10}$ E. H. Brandt, Phys. Status Solidi B 64, 467 (1974).

${ }^{11}$ D. Herlach, G. Majer, J. Major, J. Rosenkranz, M. Schmolz, W. Schwarz, A. Seeger, W. Templ, E. Brandt, U. Essmann et al., Hyperfine Interact. 63, 41 (1990).

${ }^{12}$ A. Maisuradze and A. Yaouanc, Phys. Rev. B 87, 134508 (2013).
${ }^{13}$ M. Laulajainen, F. D. Callaghan, C. V. Kaiser, and J. E. Sonier, Phys. Rev. B 74, 054511 (2006).

${ }^{14}$ J. Schelten, H. Ullmaier, and W. Schmatz, Phys. Status Solidi B 48, 619 (1971).

${ }^{15}$ R. Kahn and G. Parette, Solid State Commun. 13, 1839 (1973).

${ }^{16}$ S. Mühlbauer, C. Pfleiderer, P. Böni, M. Laver, E. M. Forgan, D. Fort, U. Keiderling, and G. Behr, Phys. Rev. Lett. 102, 136408 (2009).

${ }^{17}$ See Supplemental Material at http://link.aps.org/supplemental/ 10.1103/PhysRevB.88.140509 we determine the muon diffusion rate in the studied $\mathrm{Nb}$ sample and estimate its possible effect on a field distribution. We provide also additional details on the analysis and demonstrate an extremely low pinning.

${ }^{18} \mathrm{~A}$. Yaouanc and P. Dalmas de Réotier, Muon Spin Rotation, Relaxation, and Resonance: Applications to Condensed Matter, International Series of Monographs on Physics 147 (Oxford University Press, Oxford, 2011).

${ }^{19}$ D. K. Finnemore, T. F. Stromberg, and C. A. Swenson, Phys. Rev. 149, 231 (1966).

${ }^{20}$ S. J. Williamson, Phys. Rev. B 2, 3545 (1970). 
${ }^{21}$ N. Y. Alekseyevskiy, V. I. Nizhankovskiy, and K.-H. Berthel, Fiz. Met. Metalloved. 37, 63 (1974).

${ }^{22}$ E. H. Brandt, J. Low Temp. Phys. 73, 355 (1988).

${ }^{23}$ A. Maisuradze, R. Khasanov, A. Shengelaya, and H. Keller, J. Phys.: Condens. Matter 21, 075701 (2009).

${ }^{24}$ J. M. Delrieu, Ph.D. thesis, University of Paris-Orsay, 1974.

${ }^{25}$ H. C. Torrey, Phys. Rev. 104, 563 (1956).

${ }^{26}$ A. Seeger, Phys. Lett. A 74, 259 (1979).

${ }^{27}$ C. P. Slichter, Principles of Magnetic Resonance (Springer, Berlin, 1996), 3rd enlarged and updated ed.

${ }^{28}$ T. Yamazaki, Hyperfine Interact. 104, 3 (1997).

${ }^{29}$ W. Schwarz, E. H. Brandt, K.-P. Doring, U. Essmann, K. Furderer, M. Gladisch, D. Herlach, G. Majer, H.-J. Mundinger, H. Orth et al., Hyperfine Interact. 31, 247 (1986).

${ }^{30}$ H. Wipf, D. Steinbinder, K. Neumaier, P. Gutsmiedl, A. Magerl, and A. J. Dianoux, Europhys. Lett. 4, 1379 (1987).
${ }^{31}$ A. Grassellino, C. Beard, P. Kolb, R. Laxdal, N. S. Lockyer, D. Longuevergne, and J. E. Sonier, Phys. Rev. ST Accel. Beams 16, 062002 (2013)

${ }^{32}$ N. D. Daniilidis, S. R. Park, I. K. Dimitrov, J. W. Lynn, and X. S. Ling, Phys. Rev. Lett. 99, 147007 (2007).

${ }^{33}$ H. A. Hanson, X. Wang, I. K. Dimitrov, J. Shi, X. S. Ling, B. B. Maranville, C. F. Majkrzak, M. Laver, U. Keiderling, and M. Russina, Phys. Rev. B 84, 014506 (2011).

${ }^{34}$ A. J. Qviller, V. V. Yurchenko, Y. M. Galperin, J. I. Vestgården, P. B. Mozhaev, J. B. Hansen, and T. H. Johansen, Phys. Rev. X 2 , 011007 (2012).

${ }^{35}$ H. R. Kerchner, D. K. Christen, and S. T. Sekula, Phys. Rev. B 24, 1200 (1981).

${ }^{36} \mathrm{Here}$, factor of 2 considers integration over spin degrees of freedom.

${ }^{37}$ A. Junod, J.-L. Jorda, and J. Muller, J. Low Temp. Phys. 62, 301 (1986). 